\title{
Drug-Induced Pathomorphosis of Glioblastoma 101.8 in Wistar Rats Treated with Doxorubicin Bound to Poly(lactide-co-glycolide) Nanoparticles
}

DOI: $10.17691 / \mathrm{stm} 2018.10 .4 .12$

Received May 25, 2018

V.V. Fedoseeva, Researcher, Laboratory of Neuromorphology ${ }^{1}$;

E.A. Postovalova, Researcher, Laboratory of Inflammation Immunomorphology';

A.S. Khalansky, PhD, Leading Researcher, Laboratory of Neuromorphology';

V.A. Razzhivina, PhD, Senior Project Manager2;

S.E. Gelperina, DSc, Head of the Laboratory of Drug Delivery Systems;

O.V. Makarova, MD, Professor, Head of the Laboratory of Inflammation Immunomorphology ${ }^{1}$

${ }^{1}$ Research Institute of Human Morphology, 3 Tsurupy St., Moscow, 117418, Russia;

2LLC "Drugs Technology", 2a Rabochaya St., Khimki, Moscow Region, 141400, Russia

The aim of the investigation was to study drug-induced pathomorphosis of glioblastoma 101.8 in Wistar rats treated with different doxorubicin formulations: doxorubicin bound to polylactide nanoparticles and doxorubicin substance.

Materials and Methods. Doxorubicin bound to poly(lactic-co-glycolic acid) (PLGA) nanoparticles was used in the investigation. The study was performed on 33 male Wistar rats receiving doxorubicin (DOX) substance or doxorubicin-loaded nanoparticles (DOX-PLGA) at a dose of $1.5 \mathrm{mg} / \mathrm{kg}$ (body weight) on days 2, 5, and 8 post tumor transplantation. Lyophilized nanoparticles were resuspended in water (DOX-PLGA) or in $1 \%$ aqueous solution of poloxamer 188 (DOX-PLGA/P188) before intravenous administration. The number of mitotically dividing and dying tumor cells was counted, and the tumor cell renewal coefficient (CRC) was calculated for evaluation of pathomorphosis of glioblastoma 101.8 induced by doxorubicin, on day 14 post transplantation. The number and volume fraction of blood vessels in tumors were determined on histological sections stained with isolectin B4.

Results. On day 6 post treatment, the tumors in rats treated with all doxorubicin formulations exhibited an increase of cell death rate, decreased proliferative activity, and a lower indicator of CRC of tumor cells, compared to untreated animals. A decrease in the volume fraction and number of blood vessels in the tumors was also observed in the animals subjected to chemotherapy. According to the tumor pathomorphism parameters assessed in this study, the most effective formulation was DOX-PLGA/P188.

Key words: experimental glioblastoma; doxorubicin; PLGA nanoparticles; poloxamer 188; drug-induced pathomorphosis.

\section{Introduction}

Glioblastoma is a fast-growing and poorly differentiated brain tumor that is difficult to treat. Life expectancy of patients with glioblastoma after making the diagnosis rarely exceeds 12 months [1]. The main method of treatment is surgical removal of the tumor followed by radiotherapy, while chemotherapy plays only a supplementary role [2]. One reason for low chemotherapy efficacy in CNS tumors is the blood-brain barrier (BBB) restricting the delivery of many drugs to the brain. The BBB functions are impaired in the central areas of the tumor as newly formed vessels being imperfect in anatomical, functional and histological terms exhibit increased permeability. However, the BBB retains its functionality in the areas of infiltrative tumor growth, preventing creation of therapeutically effective drug concentrations in these areas [3]. This fact suggests significance of searching for the ways to improve the efficacy of chemotherapy for brain tumors, particularly, glioblastomas.

One promising trend in this field is the use of drug delivery systems. Biopolymer-based nanoparticles are able to deliver anti-tumor substances whose penetration across the BBB is restricted without these nanoparticles. Nanoparticles accumulate in the tumor and gradually release the encapsulated drug substance maintaining its therapeutically effective concentration [3, 4].

Doxorubicin (DOX) is an anthracycline antibiotic widely used in oncotherapy. Its molecules induce DNA damage by intercalation, inhibit proliferation or cause death in tumor cells. The cytotoxic effect of doxorubicin is attributable to the generation of free forms of oxygen and the ability to inhibit blood vessel growth in tumors [5, 6]. However, doxorubicin, a substrate of P-glycoprotein, is believed to be incapable of penetrating the BBB and therefore it can hardly be selected for chemotherapy of brain tumors. At the same time, treatment of glioblastoma

Corresponding author: Vera V. Fedoseeva, e-mail: vera_mgavmb@rambler.ru 
101.8 with doxorubicin bound to polybutylcyanoacrylate (PBCA) and polylactide (PLGA) nanoparticles has been shown to enhance survival and life expectancy of animals, promote decrease in tumor volume and inhibit its growth [7-9].

Nanoparticle surface modification by poloxamer 188 (Pluronic F68, P188, etc.) improves the antitumor effect of DOX nanoparticles [8]. Poloxamers are polymer compounds, block copolymers of polyoxyethylene (hydrophobic part of the molecule) and polyoxypropylene (hydrophilic part of the molecule), the difference in their affinity for water gives poloxamers in aqueous solution the properties of surfactants. They are used as transport systems for the delivery of drugs to the affected organs and tissues [10]. Poloxamer 188 increases the solubility and permeability of various hydrophobic compounds, has a thrombolytic effect, improves blood rheology, promotes phagocytosis, integrates into cell membranes helping to eliminate their defects [11]. Upon administration of PLGA nanoparticles into the blood, the poloxamer 188 bound to their surface promotes adsorption of apolipoproteins $E$ and $A$ that interact with LDL receptors expressed on the membranes of endothelial cells forming the BBB and thus mediate the endocytosis of nanoparticles into these cells. Nanoparticles then reach the brain via transcytosis [10].

The gold standard applied to assess the antitumor efficacy of chemotherapy and radiotherapy has been a morphological study that enables predicting recurrence and survival in cancer patients when combined with morphometric and molecular biological methods [12].

To assess treatment efficacy, comparative evaluation of drug-induced pathomorphosis of glioblastoma 101.8 was performed in Wistar rats after antitumor therapy with different doxorubicin formulations: its substance or doxorubicin bound to PLGA nanoparticles.

\section{Materials and Methods}

Nanoparticles were obtained from DOX substance (Sicor S.p.A, Italy) and a copolymer of poly(lactic-coglycolic acid) (PLGA) (Resomer RG 502H; Evonik Röhm, Germany) by double emulsion method with ratio of DOX:PLGA=1:10 [4]. After removing the organic solvents, the resulting nanosuspension was lyophilized with addition of a cryoprotector ( $1 \%$ mannit). The mean diameter of the obtained particles measured by photon correlation spectroscopy (Zetasizer Nano ZS; Malvern, UK) was $\sim 100 \mathrm{~nm}$, the degree of doxorubicin inclusion was $\sim 90 \%$. Before administration to the animals, lyophilized nanoparticles were resuspended in water (DOX-PLGA) or in 1\% aqueous solution of poloxamer 188 (DOX-PLGA/P188).

The study was carried out using 33 male Wistar rats with weighing 150-200 g. Work with animals was performed in accordance with order No. $199 \mathrm{H}$ "On the Approval of the Rules of Good Laboratory Practice" (Russia, 2016) and International Guiding Principles for Biomedical Research Involving Animals (CIOMS and ICLAS, 2012), it also complied with the Guidelines for Preclinical Trials of Medicinal Products (Mironov et al., 2012). The work was performed in accordance with ethical principles established by the European Convention for the Protection of Vertebrate Animals used for Experimental and Other Scientific Purposes (Strasburg, 2006) and was approved by Ethics Committee of Research Institute of Human Morphology.

Rat glioblastoma 101.8 has been obtained and maintained in the collection of Research Institute of Human Morphology [13]. The tumor tissue was implanted into the rat brain under anesthesia (Zoletil 100 at the dose of $6 \mathrm{mg} / 100 \mathrm{~g}$ of animal weight). The technique of tumor transplantation has been described in detail in the previous work [7].

The tumor-bearing animals were distributed into 4 groups depending on the drugs received: doxorubicin substance (DOX; $n=6)$; doxorubicin bound to PLGA nanoparticles modified by poloxamer 188 (DOX-PLGA/ P188; $n=9$ ) or unmodified ones (DOX-PLGA; $n=11$ ). Untreated animals were used as controls $(n=7)$. The drugs were injected intravenously on days 2,5 , and 8 after tumor transplantation at the dose of $1.5 \mathrm{mg} / \mathrm{kg}$.

For histological examination of glioblastoma 101.8, the animals were sacrificed on day 14 post tumor implantation (day 6 post treatment). The brain was fixed in $10 \%$ buffered formalin solution, histological sections were made and stained with hematoxylin and eosin.

To assess the proliferative potential, cell death and to calculate the tumor cell renewal coefficient of glioblastoma 101.8, the number of mitoses and dying cells was counted on the standard histological section area $\left(65,025 \mu^{2} ; x 400\right)$. In each case, counting was performed in 5-10 fields of view, depending on the size of the tumor. The tumor cell renewal coefficient (CRC) was calculated using the formula: $C R C=$ number of mitoses/number of dying cells.

Blood vessels in glioblastoma 101.8 were detected by histochemical reaction with isolectin B4 (Vector Lab, USA) to mark endothelial cells. The number of vessels in the field of view was estimated at 200 magnification. The volume fraction of blood vessels was determined by point counting method using Avtandilov's grid [14].

Statistical data processing was performed by means of Statistica 6.1 software, using non-parametric method. Median (Me) and quartile range $(25 ; 75 \%)$ served to characterize the experimental groups. One-way ANOVA test was applied to compare experimental groups, the Dunn and the Conover-Iman methods were used for multiple comparison.

\section{Results}

The morphological study of glioblastoma 101.8 in rats from different experimental groups revealed that high cell count, pronounced cellular atypia and hyperchromia of the nuclei, polymorphism in shape and 
size were characteristic of all tumors. There were a lot of tumor cells dividing by mitosis (Figure 1 (a), (b)). There were pathological mitoses: concentric mitoses, hollow metaphase, asymmetric mitoses, colchicine-like mitoses. There were many dying cells with homogeneous basophilic nucleus or nuclear fragmentation, homogeneous eosinophilic cytoplasm, shadow cells with poorly defined contours, weakly basophilic nuclei and vacuolated cytoplasm (Figure 1 (c), (d)). Infiltrative perivascular and perineural tumor growth was observed in the surrounding brain tissue: tumor cells formed clusters around vessels and dying neurons in the border zone. They often formed gland-like structures around the neurons.

A large number of blood vessels arranged in groupings in the form of vascular rosettes were revealed in all tumors (Figure 2). The vessels had deformed lumens, round or oval shape and a thin wall represented by the endothelium and a small amount of fine fibers, slit-like vessels with proliferation of the endothelium and partially formed wall were also detected (Figure 3).

Both small and extensive, single and multiple hemorrhages were often revealed in the tumors. Focal necroses were detected in many tumors, especially in those with a relatively large size, among them there were necroses with palisade-like arrangement of tumor cells. Reticular perivascular and pericellular edema was detected in the peritumoral zone. There was also detected cytolysis and death of neurons that were hyperchromic like hypoxic neurons.

The morphological features of experimental glioblastoma 101.8 revealed in the study correspond to the main features of human glioblastoma (high-grade cellular atypia, presence of numerous blood vessels, necroses and hemorrhages), which suggests similar response to treatment.
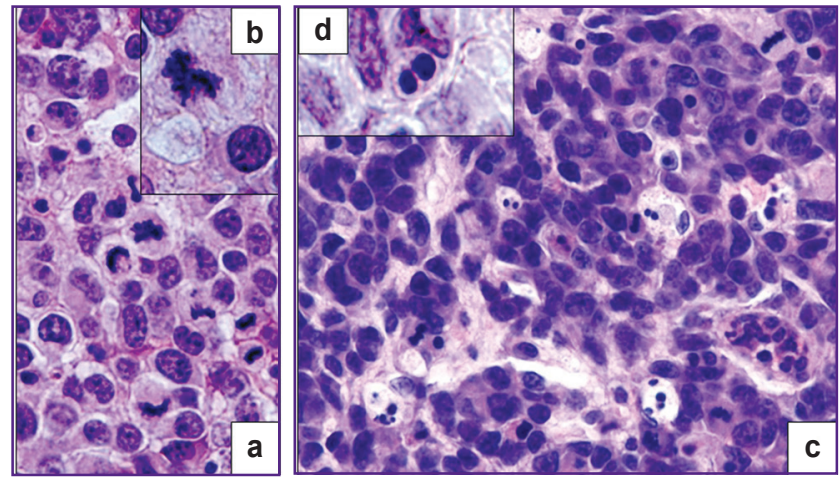

Figure 1. Morphological features of glioblastoma 101.8: the group without treatment: (a) mitotic figures; (b) multipolar mitosis; dying cells: (c) the DOX group; (d) the DOX-PLGA/ P188 group; starry-sky pattern; hematoxylin and eosin staining; magnification: (a) 400; (c) 640; (b), (d) 1000

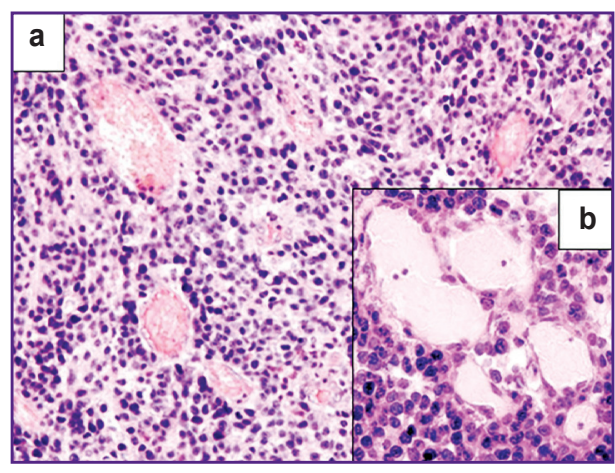

Figure 2. Morphological features of blood vessels in glioblastoma 101.8:

(a) DOX P188; thin-walled blood vessels; (b) DOX-PLGA; vascular rosette (groupings of oval-shaped thin-walled blood vessels); hematoxylin and eosin staining; magnification: (a) 400 , (b) 640

Figure 3. Histochemical reaction with isolectin B4, a marker of endothelial cells:

(a) the group without treatment, vessels with wide deformed lumens and thin walls; (b)-(d) vessels with partially formed walls and slit-like lumen: (b) DOX; (c) DOX-PLGA; (d) DOX-PLGA/P188; magnification: (a), (d) 100; (b), (c) 200
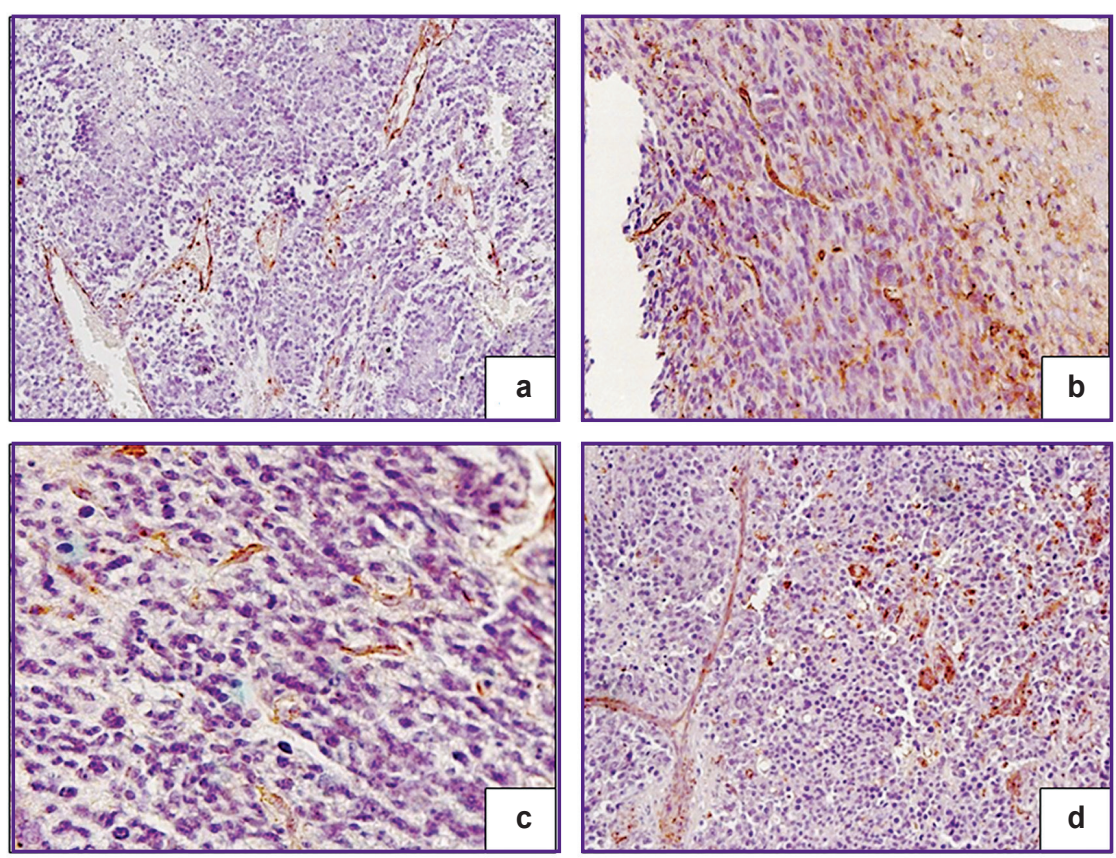

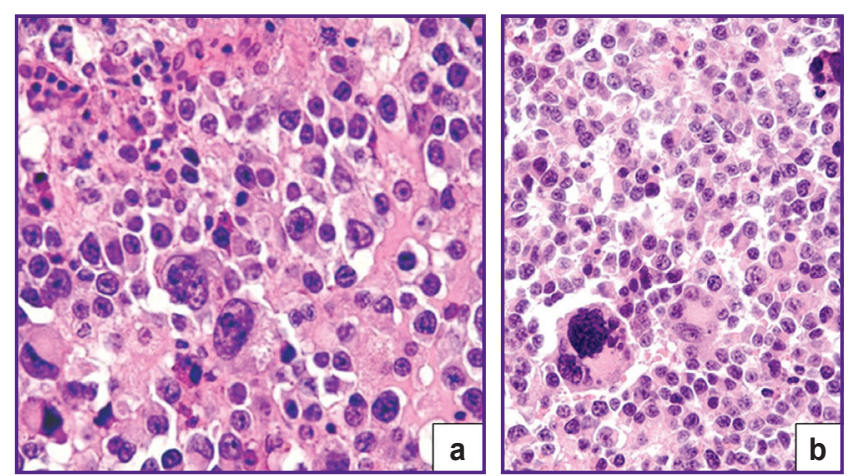

The rats from experimental groups treated with DOX, DOX-PLGA, and DOX-PLGA/P188 showed the signs of drug pathomorphosis, compared to untreated animals: tumor sizes were significantly smaller, most animals had no necroses, half of the rats had "monster" cells: from solitary cells in the preparation and the field of view to 5-7 cells in the field of view (Figure 4). Blood vessels were basically slit-like with partially formed thin walls and

Table 1

The number of cells dividing by mitosis, dying tumor cells and the tumor cell renewal coefficient (CRC) in glioblastoma-bearing rats (\%) (Me [25; 75])

\begin{tabular}{lccc}
\multicolumn{1}{c}{ Groups } & Dividing cells & Dying cells & CRC \\
\hline 1. Without treatment & $15[7 ; 11]$ & $2[1 ; 3]$ & $6.5[4.0 ; 9.5]$ \\
\hline 2. DOX & $11[9 ; 11]$ & $10.0[7.0 ; 13.5]$ & $1.04[0.78 ; 1.28]$ \\
\hline 3. DOX-PLGA & $8.0[6.0 ; 12.5]$ & $8.0[4.0 ; 14.5]$ & $1.52[0.45 ; 3.12]$ \\
4. DOX-PLGA/P188 & $5[4 ; 6]$ & $14.5[10.0 ; 17.0]$ & $0.38[0.25 ; 0.5]$ \\
\hline$p$ & $p(1-2)=0.01$ & $p(1-2)=0.00002$ & $p(1-2)=0.00001$ \\
& $p(1-3)=2.3 E$ & $p(1-3)=3.11 E$ & $p(1-3)=0.00003$ \\
& $p(1-4)=0.00002$ & $p(1-4)=0.00001$ & $p(1-4)=0.00001$ \\
& $p(2-3)=0.007$ & $p(2-3)=0.02$ & $p(2-4)=1.28 E$ \\
& $p(2-4)=2.55 E$ & $p(2-4)=0.01$ & $p(3-4)=3.37 E$ \\
& $p(3-4)=1.52 E$ & $p(3-4)=5.59 E$ & \\
\hline
\end{tabular}

Table 2

Volume fraction and number of blood vessels in rat glioblastoma 101.8 after administration of different doxorubicin formulations (\%) (Me [25; 75])

\begin{tabular}{|ccc|}
\hline \multicolumn{1}{c}{$\begin{array}{c}\text { Groups (number } \\
\text { of observations) }\end{array}$} & $\begin{array}{c}\text { Volume fraction } \\
\text { of blood vessels (volume } \%)\end{array}$ & $\begin{array}{c}\text { Number } \\
\text { of blood vessels }\end{array}$ \\
\hline 1. Without treatment $(n=171)$ & $5[3 ; 6]$ & $5[4 ; 6]$ \\
\hline 2. DOX $(n=89)$ & $1.5[0.5 ; 2.5]$ & $3[2 ; 4]$ \\
\hline 3. DOX-PLGA $(n=69)$ & $4[2 ; 6]$ & $5[4 ; 6]$ \\
\hline 4. DOX-PLGA/P188 & $2[1 ; 3]$ & $2[1 ; 3]$ \\
\hline$p$ & $p(1-2)=3 E-06$ & $p(1-2)=4 E-10$ \\
& $p(1-4)=0.003$ & $p(1-4)=0.012$ \\
& $p(2-3)=1 E-04$ & $p(2-3)=7 E-06$ \\
& $p(3-4)=0.005$ & $p(2-4)=6 E-04$ \\
& $p(3-4)=0.026$ \\
\hline
\end{tabular}

Figure 4. Morphological features of glioblastoma 101.8 cells:

(a) DOX-PLGA/P188; (b) DOX-PLGA; tumors with high cellularity of polymorphic atypical cells with hyperchromatic nuclei and narrow cytoplasmic rim; there are multinucleated monster cells among atypical cells; magnification: (a) 640, (b) 400

deformed lumens. It should be noted that the intensity of drug-induced pathomorphosis varied within each group of animals under study.

In all groups of animals receiving treatment, the number of dying tumor cells increased and mitosis rates were lower compared to those untreated. The minimum number of mitoses was observed in rats with glioblastoma treated with DOX-PLGA/P188 (Table 1).

Compared to untreated animals, the absolute number of blood vessels in tumors was reduced after treatment in almost all groups of rats. This number was the lowest in the DOX-PLGA/ P188 group, while in the group receiving DOXPLGA, it did not differ from the group without treatment.

The morphometric analysis of glioblastoma 101.8 showed that the median of vascular volume fraction decreased in all groups of treated animals. This index was low in the DOX and DOX-PLGA/P188 groups (Table 2).

Thus, the number and volume fraction of blood vessels in the tumor were significantly lower in the group of animals treated with DOX-PLGA/P188 as compared to all experimental groups.

\section{Discussion}

The revealed morphological changes in glioblastoma 101.8 under the influence of chemotherapy with DOX and its nanosomal form should be regarded as manifestations of drug-induced pathomorphosis. The issue of drug-induced pathomorphosis has been described in detail by Lushnikov [15]: pathomorphosis is both general and local manifestations of tumor growth under the influence of any action. Today, pathomorphosis (from Greek pathos - disease and morphosis - formation) is understood as persistent changes in the clinical and morphological picture of the disease under the influence of environmental factors. The problem of morphological criteria for druginduced ("medicinal", "medical", "therapeutic") 
pathomorphosis of tumors remains debatable and largely depends on tumor nature, the type of therapeutic action, the mechanism of action, the mode of drug administration, etc. The most significant parameters of pathomorphosis are as follows: tumor growth rate and character, the presence and degree of cell atypia, the ratio of stroma-parenchyma volume fractions, polymorphism or uniformity of cell shape and size, the nuclear-cytoplasmic ratio, the presence and number of mitoses, dying cells, dystrophic changes in tumor cells. It is also important to take into account the type of angiogenesis evaluated by microvascular density, the total perimeter, and area of blood vessels, the presence of necrotic and hemorrhagic foci, etc. [16-20].

There is still no standard for evaluation of druginduced pathomorphosis of human malignant brain tumors. The basis for evaluation of morphological changes in treatment of experimental glioblastoma 101.8 is the Daumas-Duport classification [21] used to predict glioblastoma progression in humans. The classification is based on four morphological features: nuclear atypia, mitotic activity, endothelial proliferation, necrosis $(0$ - absence of the sign or its doubtful manifestation; 1 - presence). Prognostic significance of the mitotic index and cell death index in tumor treatment has been confirmed by investigating numerous malignant tumors of various localizations [18, 22].

Histologic interpretation of glioblastoma 101.8 preparations of all experimental groups revealed the described signs of drug-induced pathomorphosis in most animals receiving treatment: decrease in tumor volumes, fewer necroses present. "Monster" cells were detected in many tumors in treated animals, which is considered a sign of tumor regression after chemotherapy [23].

Pathomorphosis of glioblastoma 101.8 induced by all the drugs under study also involved intensification of tumor cell death processes. Glioblastomas of animals treated with DOX-PLGA/P188 were characterized by the increased rate of tumor cell death compared to the group of rats treated with DOX.

All doxorubicin formulations reduced the proliferative activity of tumor cells and increased their death. In this regard, cell turnover rate was lower in treated animals than in those untreated.

High vascularization is characteristic of glioblastoma 101.8 and its decrease is an indicator relevant for evaluating tumor treatment efficacy [15]. The absolute number of tumor blood vessels and their volume fraction decreased in all groups of treated animals. However, the best result was observed in the group of animals treated with DOX-PLGA/P188.

The data obtained correlate with the previously described results of investigating the antitumor activity of the nanosized form of doxorubicin against glioblastoma 101.8. For example, the studies carried out by Hekmatara et al. [5], Steiniger et al. [9], Khalansky et al. [24] show that drug-induced pathomorphosis of experimental glioblastoma 101.8 in Wistar rats treated with nanosomal doxorubicin bound to PBCA nanoparticles is characterized by decrease in tumor size and vascularization degree, prevalence of necroses, decreased proliferative indices [25], reduced volume density of blood vessels and necroses in the tumor. High efficacy of treating glioblastoma 101.8 with doxorubicin bound to PLGA nanoparticles was also shown by Wohlfart et al. [26] who used semi-quantitative scoring system to evaluate the extent of necrosis and vascular rosettes.

\section{Conclusion}

All doxorubicin formulations reduce the proliferative activity of tumor cells and increase their death, thereby reducing cell turnover rate in treated animals. DOXPLGA and DOX-PLGA/P188 appear to be the most effective therapeutic compounds reducing proliferative activity and increasing tumor cell death. When different doxorubicin formulations are administered in rats, the volume fraction of blood vessels in the tumor decreases only in the groups treated with DOX and DOX-PLGA/ P188 as compared to untreated animals.

As the result of evaluating the pathomorphosis of glioblastoma 101.8 against standard morphological criteria, DOX-PLGA/P188 was found to exhibit the maximum antitumor activity. The experimental study results make it possible to consider DOX-loaded PLGA nanoparticles resuspended in $1 \%$ aqueous solution of P188 poloxamer to be the most effective antitumor agent.

Study funding. The study was performed within the frame of Federal Target Program "Development of Pharmaceutical and Medical Industries in the Russian Federation for the Period up to 2020 and the Future" (Government contract 13411.1008799.13.144).

Conflict of interests. The authors have no conflict of interests to disclose.

\section{References}

1. Johnson D.R., Omuro A.M.P., Ravelo A., Sommer N., Guerin A., Ionescu-Ittu R., Shi S., Macalalad A., Uhm J.H. Overall survival in patients with glioblastoma before and after bevacizumab approval. Curr Med Res Opin 2018; 34(5): 813820, https://doi.org/10.1080/03007995.2017.1392294.

2. Furnari F.B., Fenton T., Bachoo R.M., Mukasa A., Stommel J.M., Stegh A., Hahn W.C., Ligon K.L., Louis D.N., Brennan C., Chin L., DePinho R.A., Cavenee W.K. Malignant astrocytic glioma: genetics, biology, and paths to treatment. Genes Dev 2007; 21(21): 2683-2710, https://doi.org/10.1101/ gad.1596707.

3. Maeda H. Macromolecular therapeutics in cancer treatment: the EPR effect and beyond. J Control Release 2012; 164(2): 138-144, https://doi.org/10.1016/j.jconrel.2012.04.038.

4. Malinovskaya Y., Melnikov P., Baklaushev V., Gabashvili A., Osipova N., Mantrov S., Ermolenko Y., Maksimenko O., Gorshkova M., Balabanyan V., Kreuter J., 
Gelperina S. Delivery of doxorubicin-loaded PLGA nanoparticles into U87 human glioblastoma cells. Int J Pharm 2017; 524(1-2): 77-90, https://doi.org/10.1016/j.ijpharm.2017.03.049.

5. Hekmatara T., Bernreuther C., Khalansky A.S., Theisen A., Weissenberger J., Matschke J., Gelperina S., Kreuter J., Glatzel M. Efficient systemic therapy of rat glioblastoma by nanoparticle-bound doxorubicin is due to antiangiogenic effects. Clin Neuropathol 2009; 28(3): 153-164, https://doi.org/10.5414/npp28153.

6. Thorn C.F., Oshiro C., Marsh S., HernandezBoussard T., McLeod H., Klein T.E., Altman R.B. Doxorubicin pathways: pharmacodynamics and adverse effects Pharmacogenet Genomics 2011; 21(7): 440-446, https://doi. org/10.1097/fpc.0b013e32833ffb56.

7. Fedoseeva V.V., Khalansky A.S., Mkhitarov V.A., Tsvetkov I.S., Malinovskaya Y.A., Maksimenko O.O., Gelperina S.E., Balabanyan V.Y., Razzhivina V.A., Gorelikov P.L., Mikhailova L.P., Makarova O.V. Anti-tumor activity of doxorubicin-loaded poly(lactide-co-glycolide) nanoparticles in the experimental glioblastoma. Klinicheskaya $i$ eksperimental'naya morfologiya 2017; 2(22): 65-71.

8. Gelperina S., Maksimenko O., Khalansky A., Vanchugova L., Shipulo E., Abbasova K., Berdiev R. Wohlfart S., Chepurnova N., Kreuter J. Drug delivery to the brain using surfactant-coated poly(lactide-co-glycolide) nanoparticles: influence of the formulation parameters. Eur J Pharm Biopharm 2010; 74(2): 157-163, https://doi. org/10.1016/j.ejpb.2009.09.003.

9. Steiniger S.C., Kreuter J., Khalansky A.S., Skidan I.N., Bobruskin A.I., Smirnova Z.S., Severin S.E., Uhl R., Kock M., Geiger K.D., Gelperina S.E. Chemotherapy of glioblastoma in rats using doxorubicin-loaded nanoparticles. Int J Cancer 2004; 109(5): 759-767, https://doi.org/10.1002/ijc.20048.

10. Kreuter J. Drug delivery to the central nervous system by polymeric nanoparticles: what do we know? Adv Drug Deliv Rev 2014; 71: 2-14, https://doi.org/10.1016/j.addr.2013.08.008.

11. Moghimi S.M., Hunter A.C. Poloxamers and poloxamines in nanoparticle engineering and experimental medicine. Trends Biotechnol 2000; 18(10): 412-420, https:// doi.org/10.1016/s0167-7799(00)01485-2.

12. Westerterp M., van Westreenen H.L., Reitsma J.B., Hoekstra O.S., Stoker J., Fockens P., Jager P.L., Van EckSmit B.L., Plukker J.T., van Lanschot J.J., Sloof G.W. Esophageal cancer: CT, endoscopic US, and FDG PET for assessment of response to neoadjuvant therapy - systematic review. Radiology 2005; 236(3): 841-851, https://doi. org/10.1148/radiol. 2363041042.

13. Collection of experimental tumors of the nervous system and neural tumor cell lines. URL: http://ckp-rf.ru/usu/498710/.
14. Avtandilov G.G. Morfologiya v patologii [Morphology in pathology]. Moscow: Meditsina; 1973; 277 p.

15. Lushnikov E.F. Luchevoy patomorfoz opukholey cheloveka [Radiation pathomorphosis of human tumors]. Moscow: Meditsina; 1977; 328 p.

16. Lavnikova G.A. Some regularities of radiation pathomorphosis of human tumors and their practical use. Vestnik AMN SSSR 1976, 6: 13-19.

17. . Patologoanatomicheskaya diagnostika opukholey cheloveka [Pathoanatomical diagnosis of human tumors]. Pod red. Kraevskogo N.A., Smolyannikova A.V., Sarkisova D.S. [Kraevskiy N.A., Smolyannikov A.V., Sarkisov D.S. (editors)] Moscow: Meditsina; 1993; 560 p.

18. Staunton M.J., Gaffney E.F. Tumor type is a determinant of susceptibility to apoptosis. Am J Clin Pathol 1995, 103(3): 300-307, https://doi.org/10.1093/ajcp/103.3.300.

19. Kazantseva I.A., Gaganov L.E. The morphological parameters of cell regeneration in gastric carcinomas and their prognostic value. Arkhiv patologii 2014; 4: 3-8.

20. Takagi H., Azuma K., Tsuka T., Imagawa T., Osaki T., Okamoto Y. Antitumor effects of high-temperature hyperthermia on a glioma rat model. Oncol Lett 2014; 7(4): 1007-1010, https://doi.org/10.3892/ol.2014.1852.

21. Daumas-Duport C., Scheithauer B., O'Fallon J., Kelly P. Grading of astrocytomas. A simple and reproducible method. Cancer 1988; 62(10): 2152-2165, https://doi.org/10.1002/10970142(19881115)62:10<2152::aid-cncr2820621015>3.0.co;2-t.

22. Lushnikov E.F., Abrosimov A.Yu. Gibel' kletki (apoptoz) [Cell death (apoptosis)]. Moscow: Meditsina; 2001; 190 p.

23. Chang F., Deere H., Mahadeva U., George S. Histopathologic examination and reporting of esophageal carcinomas following preoperative neoadjuvant therapy: practical guidelines and current issues. Am J Clin Pathol 2008; 129(2): 252-262, https://doi.org/10.1309/ccr3qn4874yjdjj7.

24. Khalansky A.S., Hekmatara T., Bernreuther C., Rubtsov B.V., Kondakova L.I., Matschke J., Kreuter J., Glatzel M., Gelperina S.E., Shvets V.I. Morphological evaluation of the antitumour effect of the nanoparticle-bound doxorubicin in a rat glioblastoma model. Biofarmatsevticheskiy zhurnal 2011; 3(2): 41-50

25. Khalansky A.S., Kondakova L.I. Transplanted rat glioma 101.8. Biological characteristics. Klinicheskaya $i$ eksperimental'naya morfologiya 2013; 4: 63-69.

26. Wohlfart S., Khalansky A.S., Gelperina S., Maksimenko O., Bernreuther C., Glatzel M., Kreuter J. Efficient chemotherapy of rat glioblastoma using doxorubicinloaded PLGA nanoparticles with different stabilizers. PLoS One 2011; 6(5): e19121, https://doi.org/10.1371/journal. pone.0019121. 